\title{
From form to function
}

DNA building blocks that interact on the basis of shape rather than sequence assemble into highly dynamic and complex structures.

Perhaps the most distinctive characteristic of DNA molecules-aside from their iconic double-helical structure-is the highly specific A-to-T and G-to-C base-pairing that holds their strands together. So it is not surprising that early practitioners of 'DNA origami' have relied almost entirely on recognition between complementary oligonucleotide sequences to drive self-assembly of nucleic acid-based nanostructures.

Sequence-based assembly can yield both dynamic devices and more complex static structures, but construction of larger DNAbased 'machines' is less feasible. "If you want to make a defined, rigid structure, you have to form many DNA double helices to stabilize it," explains Hendrik Dietz of the Technische Universität München. "But once you have this many double helices, it's very hard to break them again." As an alternative, Dietz and his colleagues took a page from the protein playbook, using DNA sequences to construct topologically defined domains that assemble with each other on the basis of shape-complementarity-like the pairing between a plug and its outlet.

Self-assembly is assisted by the fact that the blunt ends of double-stranded DNA are 'sticky', in the sense that they can adhere to other DNA blunt ends via hydrophobic stacking interactions. The strength of these interactions can be modulated by environmental factors, including temperature or the concentration of positively charged ions. This feature allows Dietz's team to directly control the assembly and disassembly of their DNA-based building blocks, yielding highly dynamic DNA structures of unprecedented complexity.

To begin, the researchers designed various sets of blocks, each featuring distinctive patterns of protrusions and indentations that governed which other blocks it could assemble with. For some designs, the DNAbased building blocks could self-assemble into lengthy filaments of precisely ordered monomers. The blocks rapidly dissociated when the magnesium concentration was reduced, only to reassemble when higher cation levels were restored.
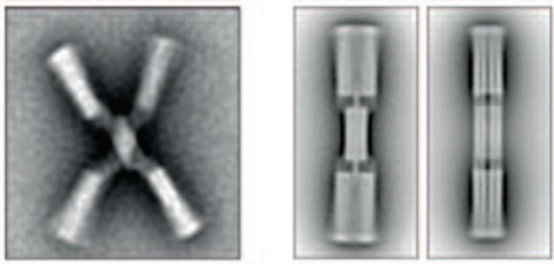

Electron micrographs of a DNA-based actuator that opens and closes in response to environmental changes. Reproduced from Gerling et al., AAAS.

Dietz and his colleagues subsequently exploited this dynamic aspect in a simple nanomachine, a scissor-like actuator that could be repeatedly induced to open and close by respectively raising and lowering the temperature. By modifying the actuator arms with a fluorophore pair, the researchers were able to use fluorescence resonance energy transfer (FRET) to directly detect the conformation change. "We found that we could do 1,020 cycles of opening and closing with no sign of degradation," says Dietz. Even more complex dynamic constructs proved feasible, including a self-assembling 'nanobot' that could raise and lower its arms and a fishnet-like mesh of actuator units that contract or expand in synchrony.

In general, Dietz reports a remarkable success rate with this technique. "You don't have to worry about the sequences needed to connect your components," he says. "It's so much easier that our new projects only use this approach." However, efficiently designing shape-complementary nanostructures will require improved modeling software-for now, his team fine-tunes their DNA designs with 3D-printed prototypes.

These proof-of-concept constructs already suggest a number of potential useful applications. For example, the FRET-coupled actuators could form the foundation for fluorescent biosensors. Drug-delivery vehicles are also a possibility, and Dietz's team has found that the elastic nature of their DNA nanostructures makes it possible to allosterically trigger conformation changes through specific binding events. "One could, for example, envision making a capsule that opens up once it has bound to a cell surface protein," says Dietz.

\section{Michael Eisenstein}

RESEARCH PAPERS

Gerling, T. et al. Dynamic DNA devices and assemblies formed by shape-complementary, nonbase pairing 3D components. Science 347, 14461452 (2015). 